JOURNAL OF

FUNCTION SPACES AND APPLICATIONS

Volume 3, Number 3 (2005), 263-286 (c) 2005, Scientific Horizon http://www.jfsa.net

\title{
Domains of pseudo-differential operators: a case for the Triebel-Lizorkin spaces
}

\section{Jon Johnsen}

(Communicated by Victor Burenkov)

2000 Mathematics Subject Classification. Primary 47G30; secondary $46 \mathrm{E} 35$.

Keywords and phrases. Type 1,1-operators, Triebel-Lizorkin spaces, Twisted diagonal, Support rule.

\footnotetext{
Abstract. The main result is that every pseudo-differential operator of type 1,1 and order $d$ is continuous from the Triebel-Lizorkin space $F_{p, 1}^{d}$ to $L_{p}$, $1 \leq p<\infty$, and that this is optimal within the Besov and Triebel-Lizorkin scales. The proof also leads to the known continuity for $s>d$, while for all real $s$ the sufficiency of Hörmander's condition on the twisted diagonal is carried over to the Besov and Triebel-Lizorkin framework. To obtain this, type 1,1-operators are extended to distributions with compact spectrum, and Fourier transformed operators of this type are on such distributions proved to satisfy a support rule, generalising the rule for convolutions. Thereby the use of reduced symbols, as introduced by Coifman and Meyer, is replaced by direct application of the paradifferential methods. A few flaws in the literature have been detected and corrected.
} 


\section{Introduction}

At first glance this article's title may seem rather unmotivated: for symbols $a$ in Hörmander's class $S_{\rho, \delta}^{d}\left(\mathbb{R}^{n} \times \mathbb{R}^{n}\right)$, ie for $a \in C^{\infty}\left(\mathbb{R}^{2 n}\right)$ such that

$$
\left|D_{\xi}^{\alpha} D_{x}^{\beta} a(x, \xi)\right| \leq C_{\alpha \beta}(1+|\xi|)^{d-\rho|\alpha|+\delta|\beta|}
$$

it is well known that for $0 \leq \delta \leq \rho \leq 1$ the operators

$$
a(x, D) u(x)=\operatorname{OP}(a) u(x)=(2 \pi)^{-n} \int e^{\mathrm{i} x \cdot \xi} a(x, \xi) \hat{u}(\xi) d \xi
$$

map the Schwartz space $\mathcal{S}\left(\mathbb{R}^{n}\right)$ continuously into itself. For $(\rho, \delta) \neq(1,1)$ the operators form a class invariant under passage to adjoints, and they extend in this way to continuous, 'globally' defined operators

$$
a(x, D): \mathcal{S}^{\prime}\left(\mathbb{R}^{n}\right) \rightarrow \mathcal{S}^{\prime}\left(\mathbb{R}^{n}\right) .
$$

But for $\rho=\delta=1$ the domain situation is different, for Ching [2] showed the existence of $a \in S_{1,1}^{0}$ such that $a(x, D)$ doesn't belong to $\mathbb{B}\left(L_{2}\left(\mathbb{R}^{n}\right)\right)$. That all operators in $\operatorname{OP}\left(S_{1,1}^{0}\right)$ are bounded on $C^{s}$ and $H^{s}$ for $s>0$ was first proved by Stein, albeit in unpublished work (cf Meyer [14] resp. Hörmander [6] for this). Continuity $H_{p}^{s+d} \rightarrow H_{p}^{s}$ for $s>0,1<p<\infty$ is due to Meyer $[14,15]$.

Bourdaud analysed adjoints of $\operatorname{OP}\left(S_{1,1}^{0}\right)$, and [1, Thm. 3] lead to criteria for a given $S_{1,1}^{0}$-operator to be bounded on $H_{p}^{s}$ for all $s \in \mathbb{R}$. For $d \in \mathbb{R}$ and $p=2$, Hörmander related this question more directly to the symbol's properties, eg via the following sufficient condition: if the partially Fourier transformed symbol $\hat{a}(\xi, \eta)=\mathcal{F}_{x \rightarrow \xi} a(x, \eta)$ vanishes in a conical neighbourhood of a non-compact part of the twisted diagonal $\{(\xi, \eta) \mid \eta=-\xi\}$, ie for some constant $C \geq 1$ fulfils

$$
\hat{a}(\xi, \eta)=0 \quad \text { for } \quad C(|\xi+\eta|+1) \leq|\eta|
$$

then $a(x, D)$ is bounded $H^{s+d} \rightarrow H^{s}$ for all $s \in \mathbb{R}$; cf [6].

However, not all symbols $a \in S_{1,1}^{d}$ fulfill (1.4) (cf [2] or (2.12) below), so it is natural to ask whether a maximal domain of definition of $a(x, D)$ exists; clearly there is no such among the $H^{s}$ with $s>0$. The next result gives affirmative answers by means of the Triebel-Lizorkin scale $F_{p, q}^{s}\left(\mathbb{R}^{n}\right)$.

Theorem. Every $a \in S_{1,1}^{d}\left(\mathbb{R}^{n} \times \mathbb{R}^{n}\right), d \in \mathbb{R}$, yields a bounded operator

$$
a(x, D): F_{p, 1}^{d}\left(\mathbb{R}^{n}\right) \rightarrow L_{p}\left(\mathbb{R}^{n}\right) \quad \text { for } \quad p \in[1, \infty[
$$




$$
a(x, D): B_{\infty, 1}^{d}\left(\mathbb{R}^{n}\right) \rightarrow L_{\infty}\left(\mathbb{R}^{n}\right)
$$

The class $\operatorname{OP}\left(S_{1,1}^{d}\right)$ contains operators $a(x, D): \mathcal{S}\left(\mathbb{R}^{n}\right) \rightarrow \mathcal{D}^{\prime}\left(\mathbb{R}^{n}\right)$, that are discontinuous when $\mathcal{S}\left(\mathbb{R}^{n}\right)$ is given the induced topology from any of the Triebel-Lizorkin spaces $F_{p, q}^{d}\left(\mathbb{R}^{n}\right)$ or Besov spaces $B_{p, q}^{d}\left(\mathbb{R}^{n}\right)$ with $p \in[1, \infty]$ and $q \in] 1, \infty]$ (while $\mathcal{D}^{\prime}$ has the usual topology).

In particular, for fixed $p \in\left[1, \infty\left[\right.\right.$, all operators in $\operatorname{OP}\left(S_{1,1}^{d}\right)$ are bounded $F_{p, 1}^{d} \rightarrow L_{p}$, but on any larger space in the $B_{p, q^{-}}^{s}$ and $F_{p, q}^{s}$-scales they will (whatever the codomain) in general only be densely defined, unbounded.

To elucidate this, note that by the results cited above there is continuity $H_{p}^{s+d} \rightarrow L_{p}$ for every $s>0$, but not in general for $s=0$. It is well known that $H_{p}^{s}=F_{p, 2}^{s}$ for $1<p<\infty, s \in \mathbb{R}$, so it could be natural to search for maximal domains among the more general Triebel-Lizorkin spaces $F_{p, q}^{d}$; here $F_{p, 1}^{d}$ is a candidate by (1.5). On the larger spaces $F_{p, q}^{d}$ with $q>1$ the theorem yields that operators in $\operatorname{OP}\left(S_{1,1}^{d}\right)$ cannot be continuous. Moreover, in the Besov scale, $B_{p, 1}^{d} \subset F_{p, 1}^{d}$ for $1 \leq p<\infty$, and also here spaces with $q>1$ are too large, in view of the theorem. In this sense the theorem is sharp for $1 \leq p<\infty$.

Remark 1.1. In $L_{p}$-theory of, say partial differential equations $H_{p}^{s}$ spaces are natural (eg $H_{p}^{s}=W_{p}^{s}$ for integer $s \geq 0$ ), but it is well known that other $L_{p}$-based scales must show up too. Eg the trace $f\left(x^{\prime}, x_{n}\right) \mapsto f\left(x^{\prime}, 0\right)$ is a surjection

$$
H_{p}^{s}\left(\mathbb{R}^{n}\right) \rightarrow B_{p, p}^{s-\frac{1}{p}}\left(\mathbb{R}^{n-1}\right) \quad \text { for } \quad s>\frac{1}{p}, 1<p<\infty
$$

hence Besov spaces are inevitable in $L_{p}$-theory of boundary problems.

Arguments in favour of Triebel-Lizorkin spaces have, perhaps, been less compelling. Although $F_{p, 2}^{s}=H_{p}^{s}$ for $1<p<\infty$, it could be argued that this need not make the $F_{p, q}^{s}$-scale a useful extension of the $H_{p}^{s}$-spaces; indeed, many properties of $F_{p, q}^{s}$ do not depend on $q$, and some technicalities would be avoided by fixing $q=2$. But the theorem shows that also $F_{p, q^{-}}^{s}$ spaces with $q=1$ are indispensable for a natural $L_{p}$-theory, also for $p=2$.

1.1. Other mapping properties. For continuity $F_{p, q}^{s+d} \rightarrow F_{p, q}^{s}$ with $s>0$, a few minor modifications of the inequalities in the theorem's proof yield estimates implying (1.8)-(1.9) below. This proof should also be interesting because Hörmander's condition (1.4) is extended to the $F_{p, q^{-}}^{s}$ and $B_{p, q}^{s}$-scales by a mere addendum to the argument for (1.8)-(1.9): 
Corollary 1.2. Every $a(x, D) \in \operatorname{OP}\left(S_{1,1}^{d}\left(\mathbb{R}^{n} \times \mathbb{R}^{n}\right)\right)$ restricts for $s>0$ and $p, q \in[1, \infty]$ to a continuous map

$$
\begin{aligned}
& a(x, D): F_{p, q}^{s+d}\left(\mathbb{R}^{n}\right) \rightarrow F_{p, q}^{s}\left(\mathbb{R}^{n}\right), \quad \text { for } \quad p<\infty, \\
& a(x, D): B_{p, q}^{s+d}\left(\mathbb{R}^{n}\right) \rightarrow B_{p, q}^{s}\left(\mathbb{R}^{n}\right) .
\end{aligned}
$$

If in addition (1.4) holds, then both (1.8) and (1.9) are valid for all $s \in \mathbb{R}$.

The corollary has a version with $p, q \in] 0, \infty]$ if only $s>\max \left(0, \frac{n}{p}-n\right)$, as accounted for in Section 6 below (this partially removes a well-known obstacle in the use of $F_{p, q}^{s}$-spaces). A more far-reaching extension result is

Proposition 1.3. Any $A$ in $\operatorname{OP}\left(S_{1,1}^{\infty}\right)$ is a map $A: \mathcal{F}^{-1} \mathcal{E}^{\prime}\left(\mathbb{R}^{n}\right) \rightarrow$ $\mathcal{S}^{\prime}\left(\mathbb{R}^{n}\right)$, with range contained in $O_{M}\left(\mathbb{R}^{n}\right)$, ie in the space of $f \in C^{\infty}\left(\mathbb{R}^{n}\right)$ fulfilling estimates $\left|D^{\alpha} f(x)\right| \leq c_{\alpha}(1+|x|)^{N_{\alpha}}$ for all $\alpha \in N_{0}^{n}$.

This shows that every type 1,1-operator is defined on a 'large' space, and that the non-extendability to $\mathcal{S}^{\prime}\left(\mathbb{R}^{n}\right)$ comes from distributions with "highfrequency oscillations" (corresponding to the fact that it is the distant part of the twisted diagonal that matters).

1.2. The methods of proof. In Sections $4-5$ below the paradifferential approach is used for the proofs of the theorem and its corollary. On the one hand, this strategy is well known and has been widely adopted for $L_{p^{-}}$ questions, eg in works of Meyer, Bui Huy Qui, Bourdaud, Marschall and Yamazaki $[14,15,16,1,11,23]$ (the list is by no means exhaustive), and here it was combined with the density of reduced symbols. This notion is due to Coifman and Meyer [3, Sec. 2.6], who in the proof of [3, Thm. 2.6.9] used it to facilitate spectral estimation of terms like $b(x, D) v$; in fact, reduced symbols have the form $b(x, \xi)=\sum_{j=0}^{\infty} m_{j}(x) \varphi\left(2^{-j} \xi\right)$ for a $C^{\infty}$ function $\varphi$ supported in a corona around the origin and a bounded set of uniformly continuous $L_{\infty}$-functions $m_{j}$, and for such symbols, inclusions of the support of $\mathcal{F}(b(x, D) v)$ into balls and annuli was easily obtained.

On the other hand, however, the combination of reduced symbols and paradifferential techniques amounts to two limit processes, which together make the action of $a(x, D)$ rather intransparent. In order to avoid this drawback, the arguments are here carried out directly on the given symbols in $S_{1,1}^{\infty}$ and distributions $u$, without recourse to density of reduced symbols or of Schwartz functions (preferable since $\mathcal{S}\left(\mathbb{R}^{n}\right)$ is not dense in eg $B_{\infty, 1}^{d}\left(\mathbb{R}^{n}\right)$ ). In doing so, the spectral estimates necessary for the paradifferential approach are now obtained by means of Proposition 1.4 below.

Among the earlier contributions, reduced symbols are also not used in $[18,13]$, but various flaws in these papers have been detected and corrected with the present work; cf Remarks 4.2 and 5.1 below. 
To explain the direct approach in more detail, it is noted that Corollary 1.2 also relies on convergence criteria for series of distributions with spectral conditions, ef Lemma 2.1 below. It is therefore essential to have control over the spectrum of $b(x, D) v$ for rather general $b$ and $v$. For $b \in \mathcal{S}\left(\mathbb{R}^{2 n}\right)$ and $v \in \mathcal{S}\left(\mathbb{R}^{n}\right)$ this can be obtained at once, since Fubini's theorem implies the well-known formula,

$$
\mathcal{F}(b(x, D) v)(\xi)=(2 \pi)^{-n} \int \hat{b}(\xi-\eta, \eta) \hat{v}(\eta) d \eta
$$

For similar purposes Hörmander [6, p. 1091] extended (1.10) to symbols $b \in \mathcal{S}^{\prime}\left(\mathbb{R}^{2 n}\right)$ with $v$ remaining in $\mathcal{S}\left(\mathbb{R}^{n}\right)$, noting that Schwartz' kernel theorem allows this (one can eg apply (1.10) to a Schwartz function first). But for the present direct treatment of symbols and distributions both in $\mathcal{S}^{\prime} \backslash \mathcal{S}$, this approach does not suffice. It is also difficult to use limiting procedures, because $\mathcal{S}$ is not dense in $B_{\infty, 1}^{d}$, eg since $v \equiv 1$ lies there; here $\hat{v}=(2 \pi)^{n} \delta_{0}$ that moreover would be demanding to make sense of in (1.10) when also $\hat{b}$ can be a singular distribution.

However, generalising a familiar convolution technique, one has the following result that, despite its classical nature, could be important for the future Littlewood-Paley analysis of pseudo-differential operators:

Proposition 1.4 (The support rule). For $b \in S_{1,1}^{\infty}\left(\mathbb{R}^{n} \times \mathbb{R}^{n}\right)$,

$$
\operatorname{supp} \mathcal{F}(b(x, D) v) \subset\{\xi+\eta \mid(\xi, \eta) \in \operatorname{supp} \hat{b}(\cdot, \cdot), \eta \in \operatorname{supp} \hat{v}\}
$$

for every $v \in \mathcal{F}^{-1} \mathcal{E}^{\prime}\left(\mathbb{R}^{n}\right)$.

Note that $v \in \mathcal{F}^{-1} \mathcal{E}^{\prime}\left(\mathbb{R}^{n}\right)$ is meaningful for $b \in S_{1,1}^{\infty}$, by Proposition 1.3, but that such $v$ 's require more than (1.10), since Proposition 1.3 contains no continuity, so that eg density arguments are difficult to use. (It is also not clear that (1.11) should follow from results about wavefront sets, for the latter only account for singularities in singular supports.) Cf Section 5.1 below for a proof that combines a convolution in $\mathcal{D}^{\prime} * \mathcal{E}^{\prime}$ on $\mathbb{R}^{2 n}$ with a trace argument.

Somewhat surprisingly, the support rule seems to be hitherto undescribed in the literature, even for classical symbols. (However, for reduced symbols (1.11) is easy to obtain, as $\mathcal{F}(b(x, D) v)$ is a finite sum of convolutions, $(2 \pi)^{-n} \sum \hat{m}_{j} *\left(\varphi\left(2^{-j} \cdot\right) \hat{v}\right)$.) At least for $b \in S_{1,1}^{\infty}$ the proposition is a novelty.

It is perhaps noteworthy that partially Fourier transformed symbols, such as $\mathcal{F}_{x \rightarrow \xi} b(x, \eta)$, enter both the support rule (1.11) and the twisted diagonal condition (1.4). This could be natural since (1.11) quite generally 
implies that the spectrum of $b(x, D) v$ cannot be larger than the combined frequencies in the symbol's $x$ - and $\eta$-dependencies.

More specifically, Proposition 1.4 has as a corollary, that if $b \in S_{1,0}^{\infty}$ and $\operatorname{supp} \hat{b} \subset K^{\prime} \times K^{\prime \prime}$ with $K^{\prime \prime} \Subset \mathbb{R}^{n}$, then

$$
\operatorname{supp} \mathcal{F}(b(x, D) v) \subset K^{\prime}+K^{\prime \prime}, \quad \text { for } \quad v \in \mathcal{S}^{\prime}\left(\mathbb{R}^{n}\right)
$$

Indeed, for $\chi \in C_{0}^{\infty}$ such that $\chi \equiv 1$ on $K^{\prime \prime}, b(x, D) v=b(x, D) \mathcal{F}^{-1}(\chi \hat{v})$ by (1.2)-(1.3), and $K^{\prime}+K^{\prime \prime}$ results from (1.11).

A brief review of the present paper has been given in [10].

Remark 1.5. Consideration of $\operatorname{OP}\left(S_{1,1}^{d}\left(\mathbb{R}^{n} \times \mathbb{R}^{n}\right)\right)$ in $F_{p, q}^{s}$-spaces was initiated by Runst [18], but unfortunately his proofs contained a flaw that one can correct by means of Proposition 1.4, cf Remark 5.1 below. Seemingly Torres [20] was the first to extend the $H_{p}^{s}$-continuity of $[14,15]$ to the $F_{p, q}^{s}$-scale, using Frazier and Jawerth's $\varphi$-transformation [4]; Torres' results are improved in two respects cf Remark 6.3 below. The case $d=s=0$ was addressed by Bourdaud [1, Thm. 1], who showed continuity $B_{p, 1}^{0} \rightarrow L_{p}$ for $1 \leq p \leq \infty$; this is a special case of the theorem since $B_{p, 1}^{0} \subset F_{p, 1}^{0}$ for $p \geq 1$.

\section{Linearisation and operators of type 1,1}

The interest in type 1,1-operators stems partly from the fact that they appear in linearisations of non-linear functions. While settling the notation, this is recalled in the present section, and it is shown that the theorem is easy to prove for operators in such linearisations.

When $F \in C^{\infty}(\mathbb{R}, \mathbb{R})$ fulfils $D^{k} F \in L_{\infty}(\mathbb{R})$ for $k \geq 1, F(0)=0$, the operator $u \mapsto F \circ u$, defined for $u \in L_{\infty}\left(\mathbb{R}^{n}, \mathbb{R}\right)$, may be written as

$$
F(u(x))=a_{u}(x, D) u(x)
$$

for some $u$-dependent $a_{u} \in S_{1,1}^{0}\left(\mathbb{R}^{n} \times \mathbb{R}^{n}\right)$. To obtain this, one may take a Littlewood-Paley decomposition $\left(\Phi_{j}\right)_{j \in \mathbb{N}_{0}}$, that is $\Phi_{j} \in C^{\infty}\left(\mathbb{R}^{n}\right)$ with $\operatorname{supp} \Phi_{j} \Subset \mathbb{R}^{n}$ (where $A \Subset B$ means that $A$ has compact closure in $B$ ) and

$$
\begin{gathered}
1 \equiv \sum_{j=0}^{\infty} \Phi_{j}(\xi) \\
j>0: \xi \in \operatorname{supp} \Phi_{j} \Longrightarrow \frac{11}{20} 2^{j} \leq|\xi| \leq \frac{13}{10} 2^{j} .
\end{gathered}
$$


Here one can set $\Phi_{j}(\xi)=\Psi_{j}(\xi)-\Psi_{j-1}(\xi)$ for $j \geq 0$, when $\Psi \in C^{\infty}(\mathbb{R}, \mathbb{R})$ is chosen such that $\Psi(t)=1$ for $t \leq \frac{11}{10}$ and $\Psi(t)=0$ for $t \geq \frac{13}{10}$ and

$$
\Psi_{j}(\xi)=\Psi\left(2^{-j}|\xi|\right), \quad \Psi_{-1} \equiv 0
$$

for pointwisely $\Phi_{0}+\cdots+\Phi_{j}=\Psi_{j} \rightarrow 1$. It is occasionally convenient to define eg $\Phi=\Phi_{j}\left(2^{j}.\right)$, which is independent of $j>0$; this extends to other situations for simplicity's sake.

Using this, and setting $v_{j}=\Phi_{j}(D) v$ and $v^{j}=\Psi_{j}(D) v$, one has $v=\sum_{j=0}^{\infty} v_{j}$ for every tempered distribution $v$, and

$$
F(u(x))=\sum_{j=0}^{\infty} m_{j}(x) u_{j}(x)
$$

with multipliers $m_{j}(x)=\int_{0}^{1} F^{\prime}\left(u^{j-1}(x)+t u_{j}(x)\right) d t$ as in [15]. It was used there that (2.5) for $u \in H_{p}^{s}$ with $s>\frac{n}{p}$ shows (2.1) for

$$
a_{u}(x, D) v(x)=\sum_{j=0}^{\infty} m_{j}(x) \Phi_{j}(D) v(x) .
$$

Clearly $a_{u}(x, \xi)=\sum_{j=0}^{\infty} m_{j}(x) \Phi_{j}(\xi)$ is its symbol; the sum is locally finite, hence $C^{\infty}$. Here $a_{u} \in S_{1,1}^{0}$, for if $j>0$ one has on $\operatorname{supp} \Phi_{j}$ that $2^{j} \doteq 1+|\xi|\left[\right.$ ie, for some $c \geq 1$, it holds that $\left.\frac{2^{j}}{c} \leq|\xi| \leq c 2^{j}\right]$, so that $D_{\xi}^{\alpha}$ on $\Phi_{j}(\xi)=\Phi\left(2^{-j} \xi\right)$ produces the factor $2^{-j|\alpha|}$, estimated by $c(1+|\xi|)^{-|\alpha|}$. Note also that $\left\|m_{j}\right\|_{\infty} \leq\left\|F^{\prime}\right\|_{\infty}$ and $\left\|D_{x}^{\beta} m_{j}\right\|_{\infty} \leq c(1+|\xi|)^{|\beta|}$.

Recall that the Triebel-Lizorkin space $F_{p, q}^{s}\left(\mathbb{R}^{n}\right)$ is defined for $s \in \mathbb{R}$, $0<p<\infty$ and $0<q \leq \infty$, as the set of $v \in \mathcal{S}^{\prime}\left(\mathbb{R}^{n}\right)$ for which

$$
\left\|v \mid F_{p, q}^{s}\right\|:=\left\|\left(\sum_{j=0}^{\infty} 2^{s j q}\left|v_{j}(\cdot)\right|^{q}\right)^{\frac{1}{q}}\right\|_{p}<\infty .
$$

This is a quasinorm for $p<1$ or $q<1$ ('quasi' will be suppressed below). Here $\|\cdot\|_{p}$ is the norm of $L_{p}\left(\mathbb{R}^{n}\right), 0<p \leq \infty$, and for $q=\infty$ the $\ell_{q^{-}}$ norm above should be replaced by an $\ell_{\infty}$-norm (this is to be understood throughout when $q=\infty$ is included). The Besov space $B_{p, q}^{s}$ is defined by taking the $L_{p}$ norm of $2^{s j} v_{j}$ first, before the $\ell_{q}$-norm. General properties of the spaces are described in [17,21] or [23]. Below it is used that $F_{p, q}^{t} \hookrightarrow F_{p, q}^{s}$ for $t>s$ and $F_{p, q}^{s} \hookrightarrow F_{p, r}^{s}$ for $0<q \leq r \leq \infty$.

For later reference, some important convergence criteria are recalled from eg [17, Prop. 2.3.2/2] or [23, Thm. 3.6-3.7], though for simplicity for $F_{p, q}^{s}$ and $p, q \in[1, \infty]$ only (cf Lemma 6.1 below). 
Lemma 2.1. Let $s>0,1 \leq p<\infty$ and $1 \leq q \leq \infty$ and suppose $\sum_{j=0}^{\infty} u_{j}$ is a series in $\mathcal{S}^{\prime}\left(\mathbb{R}^{n}\right)$ to which there exists $A>0$ such that, for $k \in \mathbb{N}_{0}$,

$$
\operatorname{supp} \mathcal{F} u_{k} \subset B\left(0, A 2^{k}\right), \quad F:=\left\|\left(\sum_{j=0}^{\infty} 2^{j s q}\left|u_{j}\right|^{q}\right)^{\frac{1}{q}}(\cdot)\right\|_{p}<\infty .
$$

Then $\sum u_{j}$ converges in $\mathcal{S}^{\prime}\left(\mathbb{R}^{n}\right)$ to some $u \in F_{p, q}^{s}\left(\mathbb{R}^{n}\right)$ and $\left\|u \mid F_{p, q}^{s}\right\| \leq c F$ for a suitable constant $c$. Moreover, if $\mathcal{F} u_{k}(\xi) \neq 0$ implies for all $k>0$, $\frac{1}{A} 2^{k} \leq|\xi| \leq A 2^{k}$, then the result is valid for all $s \in \mathbb{R}$.

The fact that $a_{u}(x, \xi)$ from $(2.6)$ has the structure of a reduced symbol immediately yields a simple version of the theorem:

Proposition 2.2. For $u \in L_{\infty}\left(\mathbb{R}^{n}, \mathbb{R}\right)$ with $a_{u} \in S_{1,1}^{0}\left(\mathbb{R}^{n} \times \mathbb{R}^{n}\right)$ defined as above, the associated operator is bounded, for every $p \in[1, \infty[$,

$$
a_{u}(x, D): F_{p, 1}^{0}\left(\mathbb{R}^{n}\right) \rightarrow L_{p}\left(\mathbb{R}^{n}\right),
$$

with $L_{p}$-convergence of (2.6) for every $v \in F_{p, 1}^{0}\left(\mathbb{R}^{n}\right)$.

Proof. For $v \in F_{p, 1}^{0}$, one finds for any finite sum

$$
\left\|\sum m_{j} \Phi_{j}(D) v\right\|_{p} \leq\left\|\sum\left|m_{j}\right| \cdot\left|v_{j}\right|\right\|_{p} \leq\left\|F^{\prime}\right\|_{\infty}\left\|\sum\left|v_{j}\right|\right\|_{p},
$$

so it follows, with $\sum_{j=0}^{\infty}\left|v_{j}(x)\right|$ as a majorant, that the series for $a_{u}(x, D) v(x)$ is fundamental in $L_{p}\left(\mathbb{R}^{n}\right)$, hence convergent. $a_{u}(x, D) v$ being defined thus, the above estimate may be read verbatim for the sum over all $j \geq 0$, which yields the claimed boundedness.

The series defining $a_{u}(x, D)$ converges in $L_{p}$, as shown, and it extends $\operatorname{OP}\left(a_{u}\right)$ defined on $\mathcal{S}\left(\mathbb{R}^{n}\right)$ by (1.2). Indeed, since $\left|a_{u}\right| \leq\left\|F^{\prime}\right\|_{\infty}$, passage to a subsequence (if necessary) and majorisation gives a.e., for $v \in \mathcal{S}\left(\mathbb{R}^{n}\right)$,

$$
a_{u}(x, D) v(x)=\lim _{N \rightarrow \infty} \int \frac{e^{\mathrm{i} x \cdot \xi}}{(2 \pi)^{n}}\left(\sum_{j=0}^{N} m_{j} \Phi_{j}\right) \hat{v} d \xi=\mathrm{OP}\left(a_{u}\right) v(x)
$$

For $v \in B_{\infty, 1}^{0}\left(\mathbb{R}^{n}\right)$, formula (2.10) holds with $p=\infty$, so by the triangle inequality $a_{u}(x, D): B_{\infty, 1}^{0} \rightarrow L_{\infty}$ is bounded. This gives (2.1) for $u$ in the subspace $B_{\infty, 1}^{0} \subset L_{\infty}\left(\mathbb{R}^{n}, \mathbb{R}\right)$. (Restriction to the diagonal will extend $(u, v) \mapsto a_{u}(x, D) v$ to all $u$ in $L_{\infty}\left(\mathbb{R}^{n}, \mathbb{R}\right) ;$ cf. (2.5).)

The counterexample needed for the theorem is essentially the same as Ching's construction [2] (that was also analysed in $[1,6]$ ); with a few 
convenient modifications this is obtained by letting

$$
a(x, \xi)=\sum_{j=1}^{\infty} 2^{j d} \Phi_{j}(\xi) e^{-\mathrm{i} x_{n} 2^{j}} .
$$

This is in $S_{1,1}^{d}$, since $2^{j} \doteq 1+|\xi|$ on the support of $\Phi_{j}=\Phi\left(2^{-j} \cdot\right)$.

Lemma 2.3. For $d \in \mathbb{R}$ there exist symbols $a \in S_{1,1}^{d}\left(\mathbb{R}^{n} \times \mathbb{R}^{n}\right)$ and functions $\theta_{N} \in \mathcal{S}\left(\mathbb{R}^{n}\right)$ such that, for all $t \in[1, \infty]$, all $\left.\left.q \in\right] 1, \infty\right]$,

$$
\theta_{N} \rightarrow 0 \text { in } F_{t, q}^{d}\left(\mathbb{R}^{n}\right)(\text { for } t<\infty) \text { and in } B_{t, q}^{d}
$$

while $a(x, D) \theta_{N} \nrightarrow 0$ in $\mathcal{D}^{\prime}\left(\mathbb{R}^{n}\right)$ for $N \rightarrow \infty$.

Proof. Take $\theta \in \mathcal{S}\left(\mathbb{R}^{n}\right) \backslash\{0\}$ with $\operatorname{supp} \hat{\theta} \subset\left\{|\xi| \leq \frac{1}{20}\right\}$ and let

$$
\hat{\theta}_{N}(\xi)=\sum_{j=N}^{N^{2}} \frac{2^{-j d}}{j} \hat{\theta}\left(\xi-2^{j} e_{n}\right)=\mathcal{F}\left(\theta \sum_{j=N}^{N^{2}} \frac{e^{i 2^{j} x_{n}}}{j 2^{j d}}\right) .
$$

Since $\Phi_{j} \equiv 1$ on $\operatorname{supp} \mathcal{F}\left(\theta e^{\mathrm{i} x_{n} 2^{j}}\right)$, any $q>1$ gives for $N \rightarrow \infty$

$$
\left\|\theta_{N} \mid F_{t, q}^{d}\right\|=\left\|\left(\sum_{j=N}^{N^{2}} j^{-q}|\theta|^{q}\right)^{\frac{1}{q}}\right\|_{t} \leq\|\theta\|_{t}\left(\sum_{N}^{\infty} j^{-q}\right)^{\frac{1}{q}} \searrow 0 .
$$

The Besov case is analogous. Because $\theta_{N}$ is defined by a finite sum, a direct computation gives for the above $a(x, \xi)$

$$
a(x, D) \theta_{N}=\left(\frac{1}{N}+\cdots+\frac{1}{N^{2}}\right) \theta(x) .
$$

Any $\varphi \in C_{0}^{\infty}\left(\mathbb{R}^{n}\right)$ with $\langle\theta, \varphi\rangle=1$ yields $\left\langle a(x, D) \theta_{N}, \varphi\right\rangle \geq \log N$, so clearly $a(x, D) \theta_{N}$ does not tend to zero in the distribution sense.

Clearly $\hat{a}(\xi, \eta)=\sum_{j=1}^{\infty}(2 \pi)^{n} 2^{j d} \delta_{-2^{j} e_{n}}(\xi) \Phi_{j}(\eta)$, so it is very visible that the condition in (1.4) on the twisted diagonal is unfulfilled. However, it is also noteworthy that $a(x, D)$ moves all frequency contributions in $\theta_{N}$ to a neighbourhood of the origin, cf (2.16); this is achieved by means of the exponentials $e^{\mathrm{i} x \cdot\left(2^{j} e_{n}\right)}$ in $a(x, \xi)$.

\section{On the definition of pseudo-differential operators}

Recall first that $a(x, D) u$ is defined for arbitrary symbols $a \in \mathcal{S}^{\prime}\left(\mathbb{R}^{2 n}\right)$ if one is content with having $u \in \mathcal{S}\left(\mathbb{R}^{n}\right)$. This is via the distribution kernel 
$K(x, y)=\left.\mathcal{F}_{\xi \rightarrow z}^{-1} a(x, \xi)\right|_{z=x-y}$,

$$
a(x, D) u(x)=\langle K(x, \cdot), u\rangle, \quad \text { for } \quad u \in \mathcal{S}\left(\mathbb{R}^{n}\right) ;
$$

this is just two designations of the functional $\varphi \mapsto\left\langle K_{j, k}, \varphi \otimes u\right\rangle, \varphi \in \mathcal{S}$. And if, say $a \in S_{1,0}^{\infty}\left(\mathbb{R}^{n} \times \mathbb{R}^{n}\right)$ suffices, $a(x, D) u$ is defined for all $u$ in $\cup H^{s}$ or $\mathcal{S}^{\prime}\left(\mathbb{R}^{n}\right)$; cf (1.3). The theorem deals with cases between these two extremes, so it is desirable to explicate how $a(x, D) u$ should be read for $a \in S_{1,1}^{d}\left(\mathbb{R}^{n} \times \mathbb{R}^{n}\right)$ and $u \in F_{p, 1}^{d}\left(\mathbb{R}^{n}\right)$.

3.1. Paradifferential techniques. Along with $u_{j}=\Phi_{j}(D) u$ it is useful to introduce the auxiliary functions $\tilde{\Phi}_{j}=\Phi_{j-1}+\Phi_{j}+\Phi_{j+1}$ and set

$$
a_{j, k}(x, \eta)=\mathcal{F}_{\xi \rightarrow x}^{-1}\left(\Phi_{j} \hat{a}(\cdot, \eta)\right) \tilde{\Phi}_{k}(\eta) .
$$

One can then make the ansatz

$$
a(x, D) u(x)=a^{(1)}(x, D) u(x)+a^{(2)}(x, D) u(x)+a^{(3)}(x, D) u(x),
$$

when the pair $(a, u)$ is such that the following series converge in $\mathcal{D}^{\prime}\left(\mathbb{R}^{n}\right)$ :

$$
\begin{aligned}
& a^{(1)}(x, D) u=\sum_{k=2}^{\infty} \sum_{j=0}^{k-2} a_{j, k}(x, D) u_{k}, \\
& a^{(2)}(x, D) u=\sum_{\substack{k=0 \\
j}}^{\infty} \sum_{\substack{j=0,1 \\
j+l \leq 1}} a_{k-j, k-l}(x, D) u_{k-l}, \\
& a^{(3)}(x, D) u=\sum_{j=2}^{\infty} \sum_{k=0}^{j-2} a_{j, k}(x, D) u_{k} .
\end{aligned}
$$

The reason for the insertion of $\tilde{\Phi}_{k}$ above is that its compact support yields $a \in S_{1,1}^{\infty} \Longrightarrow a_{j, k} \in S^{-\infty}$, so all terms $a_{j, k}(x, D) u_{k}$ make sense for $u \in \mathcal{S}^{\prime}$.

Clearly $\tilde{\Phi}_{k}$ is redundant in $a_{j, k}(x, D) u_{k}$, for $\tilde{\Phi}_{k} \Phi_{k} \equiv \Phi_{k}$ applies in (1.2) for $u \in \mathcal{S}$, so that $\mathcal{S}^{\prime}$-continuity gives

$$
a_{j, k}(x, D) u_{k}=\operatorname{OP}\left(\Phi_{j}\left(D_{x}\right) a(x, \xi) \Phi_{k}(\xi)\right) u, \quad u \in \mathcal{S}^{\prime} .
$$

It is also convenient eg for the later application of Proposition 4.1 below to have compact support in $\xi$ of $a_{j, k}$. 
Note also that when $K_{j, k}$ denotes the kernel of $a_{j, k}(x, D)$, there is a specific meaning of $(3.1)$ applied to $a_{j, k}(x, D)$, namely the integral

$$
a_{j, k}(x, D) u_{k}=\int_{\mathbb{R}^{n}} K_{j, k}(x, y) u_{k}(y) d y, \quad \text { for } \quad u \in \mathcal{S}^{\prime}\left(\mathbb{R}^{n}\right)
$$

Indeed, since $\operatorname{supp} a_{j, k}(x, \cdot) \Subset \mathbb{R}^{n}$, the Paley-Wiener-Schwartz Theorem and the inequality $(1+|y|)^{N} \leq c_{N}(1+|x|)^{N}(1+|y-x|)^{N}$ yield that $K_{j, k}(x, \cdot)$ is $\mathcal{O}\left((1+|y|)^{N}\right)$ for any $N<0$, while $u_{k}(y)$ is so for an $N \geq 0$, whence the integral exists; (3.8) follows if $u_{k} \in \mathcal{C}_{0}^{\infty}\left(\mathbb{R}^{n}\right)$ from Fubini's theorem, so one can insert $\Psi_{m} u_{k}$ in (3.8) and let $m \rightarrow \infty$.

The $a^{(j)}$-series are thus well defined, and they converge if $a \in S_{1,1}^{\infty}$ and $u \in \mathcal{S}$; this follows from the proof of the theorem in Section 4.2 below. Granted this convergence, $a(x, D) u$ defined in (3.3) is easily seen to equal $\mathrm{OP}(a) u$ : indeed, using (3.7) and majorised convergence for $\varphi \in C_{0}^{\infty}$,

$$
\begin{aligned}
\left\langle\sum_{j=1}^{3} a^{(j)}(x, D) u, \varphi\right\rangle & =\lim _{N \rightarrow \infty}\left\langle\operatorname{OP}\left(\sum_{j=0}^{N} \Phi_{j}\left(D_{x}\right) a\right)\left(\sum_{k=0}^{N} u_{k}\right), \varphi\right\rangle \\
& =\lim _{N \rightarrow \infty} \int \frac{e^{\mathrm{i} x \cdot \xi}}{(2 \pi)^{n}} \varphi(x)\left(\Psi_{N} \hat{u}\right)(\xi) \Psi_{N}\left(D_{x}\right) a(x, \xi) d(x, \xi) \\
& =\langle\operatorname{OP}(a) u, \varphi\rangle .
\end{aligned}
$$

Therefore any continuity result proved for $a(x, D)$, with $a \in S_{1,1}^{d}$, constitutes an extension of $\operatorname{OP}(a): \mathcal{S} \rightarrow \mathcal{S}$, in a unique way when $\mathcal{S}$ is dense. This will be the case for the extension to $F_{p, 1}^{d}$ with $1 \leq p<\infty$ obtained in Section 4.2 below. But eg for $u \in B_{\infty, 1}^{d}$ the paradifferential ansatz above not only describes but also defines the distribution $a(x, D) u$.

It is important, and essentially known, that the procedure above gives back the usual pseudo-differential operators, but in lack of a reference a proof is supplied:

Lemma 3.1. If $a \in S_{1,0}^{\infty}\left(\mathbb{R}^{n} \times \mathbb{R}^{n}\right)$ and $u \in \mathcal{S}^{\prime}\left(\mathbb{R}^{n}\right)$ the series in (3.4)(3.6) converge in $\mathcal{S}^{\prime}\left(\mathbb{R}^{n}\right)$, and (3.3) gives a $(x, D) u$ as defined by (1.2) ff.

Proof. With $(\cdot \mid \cdot)$ denoting sesqui-linear duality, $a(x, D) u$ is the functional $\mathcal{S} \ni \varphi \mapsto(u \mid b(x, D) \varphi)$ for all $u \in \mathcal{S}^{\prime}$ when $a \in S_{1,0}^{d}$ is given and $b(x, \xi)=\exp \left(\mathrm{i} D_{x} \cdot D_{\xi}\right) \bar{a}(x, \xi)$. It may be seen as in [5, Thm. 18.1.7 $\mathrm{ff}]$ that $a \mapsto b$ is continuous in $S_{1,0}^{d}$, and this applies to $a^{(1)}(x, D)$, that by (3.7) has the symbol

$$
a^{(1)}(x, \xi)=\sum_{k=2}^{\infty} \Psi_{k-2}\left(D_{x}\right) a(x, \xi) \Phi_{k}(\xi) .
$$


Indeed, this series converges to $a^{(1)}(x, \xi)$ in the topology of $S_{1,0}^{d+1}$, so

$$
\begin{aligned}
\left(\sum_{j+2 \leq k \leq N} a_{j, k}(x, D) u \mid \varphi\right) & =\sum_{k=2}^{N}\left(u \mid\left(e^{\mathrm{i} D_{x} \cdot D_{\xi}}\left(\Psi_{k-2}\left(D_{x}\right) \bar{a} \Phi_{k}\right)\right)(x, D) \varphi\right) \\
& \underset{N \rightarrow \infty}{\longrightarrow}\left(u \mid\left(e^{\mathrm{i} D_{x} \cdot D_{\xi}}\left(\bar{a}^{(1)}(x, \xi)\right)\right)(x, D) \varphi\right) .
\end{aligned}
$$

Here the continuous dependence of the symbol in (1.2) was also used. Similarly the series for $a^{(2)}$ and $a^{(3)}$ converge, so the right hand side of (3.3) has an action on $\varphi$ equal to $\left(u \mid \exp \left(\mathrm{i} D_{x} \cdot D_{\xi}\right) a(x, D) \varphi\right)$, ie $(a(x, D) u \mid \varphi)$.

Remark 3.2. When (3.3) $\mathrm{ff}$ is applied to $a_{u}(x, \xi)$ from Proposition 2.2, the theorem gives boundedness $a_{u}(x, D): F_{p, 1}^{0} \rightarrow L_{p}$, but this equals the operator in Proposition 2.2 in view of $(2.11),(3.9)$ and the density of $\mathcal{S}$ in $F_{p, 1}^{0}$. (The case $B_{\infty, 1}^{0}$ seems to require another treatment.)

3.2. Proof of Proposition 1.3. Following the approach above, one can show that for $\operatorname{supp} \mathcal{F} u \Subset \mathbb{R}^{n}, a \in S_{1,1}^{\infty}$, the series $a^{(1)}(x, D) u, a^{(2)}(x, D) u$ and $a^{(3)}(x, D) u$ all converge (the first two are finite sums, and for $a^{(3)}$ one may sum over $j<N$ in (3.8) and let $N \rightarrow \infty)$. But there is an equivalent more transparent method, giving directly that the range is in $O_{M}\left(\mathbb{R}^{n}\right)$.

If $\hat{u} \in \mathcal{E}^{\prime}, a \in S_{1,1}^{\infty}$ and $\chi \in C_{0}^{\infty}$ equals 1 in a compact neighbourhood of $\operatorname{supp} \hat{u}$, then $b(x, \xi)=a(x, \xi) \chi(\xi)$ is in $S^{-\infty}$, hence $f:=b(x, D) u \in$ $O_{M}$ (that $\operatorname{OP}\left(S^{-\infty}\right)$ maps $\mathcal{S}^{\prime}$ to $O_{M}$ is proved in eg [19]). If $\tilde{\chi}$ is another such cut-off function and $\tilde{b}$ the corresponding symbol in $S^{-\infty}$, then $(b-\tilde{b})(x, D) v=0$, for a convolution of $\hat{u}$ with a sequence of $C_{0}^{\infty}$ functions may produce a sequence $\varphi_{k} \in \mathcal{S}$ that tends to $u$ in $\mathcal{S}^{\prime}$ while $(b-\tilde{b})(x, D) \varphi_{k}=0$ eventually.

Moreover, $f=\operatorname{OP}(a) u$ if $u \in \mathcal{F}^{-1} C_{0}^{\infty}=\mathcal{S} \cap \mathcal{F}^{-1} \mathcal{E}^{\prime}$; and $f=$ $a(x, D) u$ if $a \in S_{1,0}^{\infty}$. Hence $a(x, D) u=f$ is unambiguously defined, and Proposition 1.3 is proved.

Note that, with the set-up of the proof above, it follows from Lemma 3.1 that the $b^{(m)}$-series converges for $m=1,2,3$. But (3.7) implies the identity

$$
b_{j, k}(x, D) u_{k}=a_{j, k}(x, D) u_{k}
$$

for all $j$ and $k$ when $\chi=1$ on a large ball, whence

$$
f=b(x, D) u=a^{(1)}(x, D) u+a^{(2)}(x, D) u+a^{(3)}(x, D) u .
$$


Thus the given definition is equivalent with the one (mentioned in the beginning of this section) that consists in proving directly that (3.4)-(3.6) all converge for $\mathcal{F} u \in \mathcal{E}^{\prime}$, hence with the one adopted in Section 4.2 below. Consequently any $A \in \mathrm{OP}\left(S_{1,1}^{d}\right)$ is well defined on the $\mathcal{S}^{\prime}$-subspace

$$
\left(\sum_{1 \leq p<\infty}^{\prime} F_{p, 1}^{d}\left(\mathbb{R}^{n}\right)\right)+B_{\infty, 1}^{d}\left(\mathbb{R}^{n}\right)+\mathcal{F}^{-1} \mathcal{E}^{\prime}\left(\mathbb{R}^{n}\right)
$$

( $\sum^{\prime}$ denotes sums with only finitely many non-trivial terms.) Indeed, if $u \in \mathcal{S}^{\prime}$ can be split according to (3.14), the calculus of limits yields that $a^{(1)}(x, D) u$ etc all converge, with limits that depend on $u$, but hence not on the splitting. Therefore $a(x, D) u$ is well defined.

\section{The general borderline case}

4.1. A pointwise estimate. To obtain the convergence of the $a^{(j)}(x, D) u$ it is convenient to use the Hardy-Littlewood maximal function

$$
M_{t} f(x)=\sup _{r>0}\left(\frac{1}{|B(x, r)|} \int_{B(x, r)}|f(y)|^{t} d y\right)^{\frac{1}{t}}, \quad 0<t<\infty .
$$

The convolution estimate $|f * g(x)| \leq c\|g\|_{\infty} \cdot M_{1} f(x)$, that clearly holds if $f \in L_{1}^{\text {loc }}$ and $g \in L_{\infty}^{\text {comp }}$, has the following extension to a 'pointwise' estimate for pseudo-differential operators, that is central for the present article.

It is remarkable that, in order to get a both weak and flexible requirement on the symbol, a homogeneous Besov norm of $b(x, \xi)$ is introduced in the $\xi$-variable, with $x$ considered as a parameter. Recall here the norm of the homogeneous Besov space $\dot{B}_{p, q}^{s}\left(\mathbb{R}^{n}\right)$,

$$
\left\|f \mid \dot{B}_{p, q}^{s}\right\|=\left(\sum_{j=-\infty}^{\infty} 2^{s j q}\left\|\varphi_{j}(D) f\right\|_{p}^{q}\right)^{\frac{1}{q}}
$$

where $1=\sum_{j=-\infty}^{\infty} \varphi_{j}$ is a partition of unity on $\mathbb{R}^{n} \backslash\{0\}$ obtained from $\varphi_{j}(\xi)=\Phi_{1}\left(2^{-j+1} \xi\right)$. The $\dot{B}_{p, q}^{s}$-norm has the dyadic scaling property:

$$
\left\|f\left(2^{k} \cdot\right)\left|\dot{B}_{p, q}^{s}\left\|=2^{k\left(s-\frac{n}{p}\right)}\right\| f\right| \dot{B}_{p, q}^{s}\right\| \quad \text { for all } k \in \mathbb{N} .
$$

Proposition 4.1 (Marschall's inequality). Let a symbol $b \in \mathcal{S}^{\prime} \cap L_{1}^{\text {loc }}$ on $\mathbb{R}^{2 n}$ and $v \in \mathcal{S}^{\prime}\left(\mathbb{R}^{n}\right)$ be such that a ball $B\left(0,2^{k}\right) \subset \mathbb{R}^{n}, k \in \mathbb{Z}$, fulfils

$$
\operatorname{supp} \mathcal{F} v \subset B\left(0,2^{k}\right), \quad \operatorname{supp} b \subset \mathbb{R}^{n} \times B\left(0,2^{k}\right) .
$$


The distribution kernel $K$ of $b(x, D)$ is then a locally integrable function, ie $K \in L_{1}^{\text {loc }}\left(\mathbb{R}^{2 n}\right) \cap \mathcal{S}^{\prime}\left(\mathbb{R}^{2 n}\right)$. Moreover, if for some $\left.\left.t \in\right] 0,1\right]$

$$
M_{t} v(x) \cdot\left\|b\left(x, 2^{k} \cdot\right) \mid \dot{B}_{1, t}^{n / t}\left(\mathbb{R}^{n}\right)\right\| \in L_{1}^{\text {loc }}\left(\mathbb{R}^{n}\right),
$$

then $K(x, \cdot) v(\cdot)$ is in $L_{1}\left(\mathbb{R}^{n}\right)$ for a.e. $x \in \mathbb{R}^{n}$, and

$$
b(x, D) v(x):=\int_{\mathbb{R}^{n}} K(x, y) v(y) d y
$$

then defines the action of $b(x, D)$ on $v$ as a function in $L_{1}^{\text {loc }}\left(\mathbb{R}^{n}\right)$ fulfilling

$$
|b(x, D) v(x)| \leq c\left\|b\left(x, 2^{k} \cdot\right) \mid \dot{B}_{1, t}^{n / t}\left(\mathbb{R}^{n}\right)\right\| M_{t} v(x)
$$

for some constant $c$ independent of $k$.

Proof. If $\psi \in C_{0}^{\infty}\left(\mathbb{R}^{2 n}\right)$ it follows from the assumption $b \in L_{1}^{\text {loc }}$ and the definition of partial Fourier transformation by duality that

$$
\begin{aligned}
\left\langle\mathcal{F}_{\xi \rightarrow z}^{-1} b, \psi\right\rangle & =(2 \pi)^{-n} \iiint e^{\mathrm{i} z \cdot \xi} b(x, \xi) \psi(x, z) d \xi d x d z \\
& =\left\langle\int \frac{e^{\mathrm{i} z \cdot \xi}}{(2 \pi)^{n}} b(x, \xi) d \xi, \psi\right\rangle .
\end{aligned}
$$

Here the last identity uses that $\int \frac{e^{\mathrm{i} z \cdot \xi}}{(2 \pi)^{n}} b(x, \xi) d \xi$ lies in $L_{1}^{\text {loc }}\left(\mathbb{R}^{2 n}\right)$, hence in $\mathcal{D}^{\prime}$. Indeed, by Fubini's theorem, $\psi(x, z) \int \frac{e^{\mathrm{i} z \cdot \xi}}{(2 \pi)^{n}} b(x, \xi) d \xi$ is integrable for any $\psi \in C_{0}^{\infty}$, and in particular if $\psi=1$ on a large ball. Hence $K(x, y)=\left.\mathcal{F}_{\xi \rightarrow z}^{-1} b(x, \xi)\right|_{z=x-y}$ is in $L_{1}^{\text {loc }}$.

It is now clear that $K(x, y) v(y)$ is measurable, so the following estimates make sense and, post festum, prove the integrability in view of (4.5). Indeed, note first that since $\mathcal{F}$ sends every convolution in $\mathcal{S}^{\prime} * \mathcal{E}^{\prime}$ into a product, cf [5, Thm. 7.1.15], it holds, since $\mathcal{F}^{-1}=(2 \pi)^{-n} \overline{\mathcal{F}}$, for a.e. fixed $x$ that $(2 \pi)^{-n} \mathcal{F}^{-1}\left(\left(e^{-\mathrm{i} x \cdot \eta} b(x,-\eta)\right) * \hat{v}\right)$ equals $K(x, \cdot) v(\cdot)$, which implies that the latter function has spectrum in $B(0,2 R)$ for $R=2^{k}$. So if $0<t \leq 1$, the Nikolskii-Plancherel-Polya inequality, cf [21, Thm. 1.4.1], gives

$$
\int|K(x, y) v(y)| d y \leq c(2 R)^{\frac{n}{t}-n}\left(\int|K(x, y) v(y)|^{t} d y\right)^{\frac{1}{t}} .
$$

Inserting $1=\sum_{j=-\infty}^{\infty} \varphi_{j}(y-x)$ a.e., for $\varphi_{j}=\Phi\left(2^{-j}.\right)$, and using that

$$
\left|K(x, y) \varphi_{j}(y-x)\right| \leq\left\|\mathcal{F}_{z \rightarrow \xi}^{-1}\left(\varphi_{j}(z) \mathcal{F}_{\xi \rightarrow z} b(x, \xi)\right)\right\|_{1}=\left\|\varphi_{j}\left(D_{\xi}\right) b(x, \cdot)\right\|_{1},
$$


the inequality (4.9) and the definition of $M_{t} v(x)$ give, in view of (4.3),

$$
\begin{aligned}
\int|K(x, y) v(y)| d y & \leq c R^{\frac{n}{t}-n}\left(\sum_{j=-\infty}^{\infty} \int_{B\left(x, 2^{j}\right)}|v(y)|^{t} d y \cdot \frac{2^{j n}}{\left|B\left(x, 2^{j}\right)\right|}\right. \\
& \left.\times\left\|K(x, \cdot) \varphi_{j}(\cdot-x)\right\|_{\infty}^{t}\right)^{\frac{1}{t}} \\
& \leq c R^{\frac{n}{t}-n}\left\|b(x, \cdot) \mid \dot{B}_{1, t}^{n / t}\right\| M_{t} v(x) \\
& =c\left\|b(x, R \cdot) \mid \dot{B}_{1, t}^{n / t}\right\| M_{t} v(x) .
\end{aligned}
$$

If the integrability is exploited to define $b(x, D) v$ by (4.6), then (4.7) holds by (4.11) and it follows from (4.5) and the observed measurability that one gets a distribution in this way. Note that by (4.6) this definition is consistent with the case in which $b \in S_{1,0}^{\infty}$, hence also if $b \in S_{1,1}^{\infty}$.

Remark 4.2. Proposition 4.1 requires detailed comments because of overlap with [13, Prop. 5(a)]. On the one hand, the estimate (4.7) is to my knowledge an original contribution of Marschall; it appeared already in his thesis [11, p. 37], albeit without details.

On the other hand, [13, Prop. 5(a)] is difficult to follow. For one thing this is because of a vague formulation requiring, in addition to (4.4), $b$ to be "a symbol $\mathbb{R}^{n} \times \mathbb{R}^{n} \rightarrow \mathbb{C}$ " (replaced by $b \in L_{1}^{\text {loc }} \cap \mathcal{S}^{\prime}$ in Prop. 4.1). Secondly his proposition is "singled out" after the proof of Proposition 4 there, where the set-up is different and it furthermore seems to be taken for granted that $b(x, D) v$ has been defined as in (4.6) (neither (4.5) nor (4.6) was mentioned in [13]); the question of finding conditions assuring that $b(x, D) v \in \mathcal{D}^{\prime}\left(\mathbb{R}^{n}\right)$ was also not treated, and all in all the situation is rather more delicate than what [13] gives reason to believe. On these grounds, the details in Proposition 4.1 and its proof should be well motivated.

4.2. Proof of the theorem. Recall first the Fefferman-Stein inequality that the maximal function in (4.1) for $1 \leq p<\infty, 1 \leq q \leq \infty$, and any $t \in] 0,1[$, satisfies

$$
\left\|\left(\sum_{k=0}^{\infty}\left|M_{t} f_{k}\right|^{q}\right)^{\frac{1}{q}}\right\|_{p} \leq c\left\|\left(\sum_{k=0}^{\infty}\left|f_{k}\right|^{q}\right)^{\frac{1}{q}}\right\|_{p} .
$$

For $u \in F_{p, 1}^{d}$ and $f_{k}:=2^{k d} u_{k}$, the right hand side equals $c\left\|u \mid F_{p, q}^{d}\right\|$. Taking a fixed $t<1$ such that $\frac{n}{t}<n+1$, this inequality together with Proposition 4.1 will essentially yield the proof of the theorem.

In addition to (4.7), further estimates of $a(x, \xi)$ follow from the natural embeddings $W_{1}^{n+1} \hookrightarrow B_{1, \infty}^{n+1} \hookrightarrow \dot{B}_{1, t}^{n / t}$ : clearly $2^{k d} \doteq\left(1+\left|2^{k} \eta\right|\right)^{d}$ on $\operatorname{supp} \tilde{\Phi}$, 
since $\frac{1}{4} \leq|\eta| \leq 4$, and since $\Psi_{k}=\Phi_{0}+\cdots+\Phi_{k}$,

$$
\begin{aligned}
\left\|\sum_{j=0}^{k-2} a_{j, k}\left(x, 2^{k} \cdot\right) \mid \dot{B}_{1, t}^{n / t}\right\| & \leq \sum_{|\alpha| \leq n+1}\left\|D_{\xi}^{\alpha}\left(\Psi_{k-2}\left(D_{x}\right) a\left(x, 2^{k+2} \cdot\right) \tilde{\Phi}\right) \mid L_{1, \xi}\right\| \\
& \leq c\left\|\tilde{\Phi} \mid W_{1}^{n+1}\right\|\|\check{\Psi}\|_{1} 2^{k d} \mu_{0, n+1}(a),
\end{aligned}
$$

with $\mu_{l, m}(a)=\sup _{x, \xi ;|\beta| \leq l,|\alpha| \leq m}(1+|\xi|)^{-(d-|\alpha|+|\beta|)}\left|D_{x}^{\beta} D_{\xi}^{\alpha} a(x, \xi)\right|$ as the seminorms defining the topology on $S_{1,1}^{d}$. Using (4.7) for each summand in $a^{(1)}(x, D) u$, the above estimate yields for $k$ in any subset of $\mathbb{N}$,

$$
\begin{aligned}
\left\|\sum_{k} \sum_{j=0}^{k-2} a_{j, k}(x, D) u_{k}\right\|_{p}^{p} \leq & \int\left|\sum_{k} 2^{k d} M_{t} u_{k}(x)\right|^{p} d x \\
& \times\left(\sup _{x, k} 2^{-k d}\left\|\sum_{j=0}^{k-2} a_{j, k}\left(x, 2^{k+2} \cdot\right) \mid \dot{B}_{1, t}^{n / t}\right\|\right)^{p} \\
\leq & c \mu_{0, n+1}(a)^{p} \int\left(\sum_{k} 2^{k d}\left|u_{k}(x)\right|\right)^{p} d x
\end{aligned}
$$

It follows that the series defining $a^{(1)}(x, D) u$ is fundamental in $L_{p}$ when $u \in F_{p, 1}^{d}\left(\mathbb{R}^{n}\right)$ for $1 \leq p<\infty$, and the same estimate with $k \in \mathbb{N}$ then gives, for $m=1$,

$$
\left\|a^{(m)}(x, D) u\right\|_{p} \leq c \mu_{0, n+1}(a)\left\|u \mid F_{p, 1}^{d}\right\| .
$$

The sum $\sum_{j=0}^{k-2}$ may now be replaced by the one pertinent for $a^{(2)}$, and then essentially the same argument yields (4.15) for $m=2$.

To handle $a^{(3)}$, note that $0=\int \breve{\Phi}_{j}(y) y^{\alpha} d y$ for any multiindex $\alpha$ and $j \geq 1$, so that Taylor's formula for $a\left(x-y, 2^{k} \xi\right)$, with $\xi$ fixed, gives

$$
\begin{aligned}
\Phi_{j}\left(D_{x}\right) a\left(x, 2^{k} \xi\right)=\sum_{|\alpha|=N} \frac{N}{\alpha !} \int(-y)^{\alpha} \check{\Phi}_{j}(y) \\
\quad \times \int_{0}^{1}(1-\tau)^{N-1} \partial_{x}^{\alpha} a\left(x-\tau y, 2^{k} \xi\right) d \tau d y
\end{aligned}
$$


The factor $(-y)^{\alpha}$ can absorb a scaling by $2^{j N}$, since $\check{\Phi}_{j}(y)=2^{j n} \check{\Phi}\left(2^{j} y\right)$ is a Schwartz function, so by the same embeddings as before

$$
\begin{aligned}
2^{j N-k d} & \left\|\Phi_{j}\left(D_{x}\right) a\left(x, 2^{k+2} \cdot\right) \tilde{\Phi} \mid \dot{B}_{1, t}^{n / t}\right\| \\
\leq c & \sum_{\substack{|\alpha| \leq n+1 \\
|\beta|=N}} \int\left|z^{\beta} \check{\Phi}(z)\right| d z \int^{(1+|\xi|)^{N}\left|D^{\alpha} \tilde{\Phi}\right| d \xi \cdot \mu_{N, n+1}(a)}
\end{aligned}
$$

This implies that

$$
\begin{aligned}
\left|\sum_{k=0}^{j-2} a_{j, k}(x, D) u_{k}(x)\right| & \leq \sum_{k=0}^{j-2}\left\|a_{j, k}\left(x, 2^{k+2} \cdot\right) \mid \dot{B}_{1, t}^{n / t}\right\| M_{t} u_{k}(x) \\
& \leq c \mu_{N, n+1}(a) 2^{-j N} \sum_{k=0}^{j-2} 2^{k d} M_{t} u_{k}(x)
\end{aligned}
$$

Combining this and (4.12) with $\sum_{j=0}^{\infty} 2^{s j q}\left(\sum_{k=0}^{j}\left|b_{j}\right|\right)^{q} \leq c \sum_{j=0}^{\infty} 2^{s j q}\left|b_{j}\right|^{q}$, which for $s<0,0<q \leq \infty$, holds for all numbers $b_{j}$, cf [23, Lem. 3.8],

$$
\begin{aligned}
\left\|\sum_{j} \sum_{k=0}^{j-2} a_{j, k}(x, D) u_{k}\right\|_{p} & \leq c \mu_{N, n+1}(a)\left\|\sum_{j} 2^{-j N}\left(\sum_{k=0}^{j} 2^{k d} M_{t} u_{k}\right)\right\|_{p} \\
& \leq c^{\prime}\left\|\sum_{j} 2^{(d-N) j}\left|u_{j}\right|\right\|_{p}
\end{aligned}
$$

For $N=1$, say, it follows in the same way as above that the series for $a^{(3)}(x, D) u$ converges in $L_{p}$; and (4.19) implies (4.15) for $m=3$. Altogether this yields $\|a(x, D) u\|_{p} \leq c \mu_{1, n+1}(a)\left\|u \mid F_{p, 1}^{d}\right\|$.

The case $B_{\infty, 1}^{d}\left(\mathbb{R}^{n}\right)$ is analogous, and the necessary counterexamples were given in Lemma 2.3 above, so the proof of the theorem is complete.

Remark 4.3. Even for $p=2$, the above proof involves Lebesgue norms on $L_{t}$ with $0<t<1$ via $M_{t} u$ ( $t$ has to be less than the sum-exponent in $\left.F_{p, 1}^{d}\right)$.

\section{The general continuity properties}

This section is devoted to the proof of the corollary and to that of Proposition 1.4. The main thing will be to prove that the spectra of the general terms in the $a^{(j)}$-series in Section 4 fulfil

$$
\operatorname{supp} \mathcal{F}\left(\sum_{j=0}^{k-2} a_{j, k}(x, D) u_{k}\right) \subset\left\{\xi \in \mathbb{R}^{n}\left|\frac{1}{5} 2^{k} \leq\right| \xi \mid \leq \frac{5}{4} 2^{k}\right\}
$$




$$
\begin{aligned}
& \operatorname{supp} \mathcal{F}\left(\sum_{\substack{j, l=0,1 \\
j+l \leq 1}} a_{k-j, k-l}(x, D) u_{k-l}\right) \subset\left\{\xi \in \mathbb{R}^{n}|| \xi \mid \leq 4 \cdot 2^{k}\right\} \\
& \operatorname{supp} \mathcal{F}\left(\sum_{k=0}^{j-2} a_{j, k}(x, D) u_{k}\right) \subset\left\{\xi \in \mathbb{R}^{n}\left|\frac{1}{5} 2^{j} \leq\right| \xi \mid \leq \frac{5}{4} 2^{j}\right\} .
\end{aligned}
$$

In addition it will be seen that if (1.4) holds, ie for some $C \geq 1$,

$$
\hat{a}(\xi, \eta)=0 \quad \text { where } \quad C(|\xi+\eta|+1) \leq|\eta|
$$

then (S2) may be supplemented by the property that for $k$ large enough, the set on the left hand side of (S2) is contained in an annulus,

$$
\operatorname{supp} \mathcal{F}\left(\sum_{\substack{j, l=0,1 \\ j+l \leq 1}} a_{k-j, k-l}(x, D) u_{k-l}\right) \subset\left\{\xi\left|\frac{1}{4 C} 2^{k} \leq\right| \xi \mid \leq 4 \cdot 2^{k}\right\} .
$$

However, granted that Proposition 1.4 holds, the inclusions (S1)-(S3) are all easy: if $\eta$ is in supp $\hat{u}_{k}$ clearly $\frac{11}{20} 2^{k} \leq|\eta| \leq \frac{13}{10} 2^{k}$, and similarly if $(\xi, \eta)$ is in the support of $\hat{a}_{j, k}=\Phi_{j}(\xi) \hat{a}(\xi, \eta) \tilde{\Phi}_{k}(\eta)$; then Proposition 1.4 gives that

$$
\xi+\eta \in \operatorname{supp} \mathcal{F} a_{j, k}(x, D) u_{k} \Longrightarrow|\xi+\eta| \leq \frac{13}{10}\left(2^{j}+2^{k}\right) .
$$

Since $j-k \in\{0, \pm 1\}$ for each $k$ in the $a^{(2)}$-series, $|\xi+\eta| \leq \frac{39}{10} 2^{k}$, so (S2) holds. (S1) and (S3) are analogous. (S2') is seen thus: given (5.1), the support rule yields for any $(\xi, \eta)$ in supp $\hat{a}_{j, k}$ so that $\xi+\eta$ is in the support of $\mathcal{F}\left(a_{k-j, k-l}(x, D) u_{k-l}\right)$, that $\eta \in \operatorname{supp} \hat{u}_{k-l}$, hence

$$
|\xi+\eta| \geq \frac{1}{C}|\eta|-1 \geq \frac{11}{20 C} 2^{k-l}-1 \geq\left(\frac{11}{40 C}-2^{-k}\right) 2^{k} ;
$$

here the right hand side is larger than $\frac{1}{4 C} 2^{k}$ for $k>3+\log _{2}(5 C)$. Combined with (S2) this shows (S2').

5.1. Proof of the support rule. Note first that for $f(t)$ in the subspace of $\mathcal{D}^{\prime}$-valued functions

$$
f \in C\left(\mathbb{R}^{n}, \mathcal{D}^{\prime}\left(\mathbb{R}^{n}\right)\right) \subset \mathcal{D}^{\prime}\left(\mathbb{R}^{2 n}\right)
$$

there is a natural trace at $t=0$ given by $f(0) \in \mathcal{D}^{\prime}\left(\mathbb{R}^{n}\right)$. Moreover, such $f$ act on $\varphi \in C_{0}^{\infty}\left(\mathbb{R}^{2 n}\right)$ by integration, ie if points in $\mathbb{R}^{2 n}$ are written $(t, x)$ 
for $t, x \in \mathbb{R}^{n}$,

$$
\langle f, \varphi\rangle=\int_{\mathbb{R}^{n}}\langle f(t), \varphi(t, \cdot)\rangle_{\mathbb{R}^{n}} d t
$$

This elementary fact may be seen as in [9, Proposition 3.5].

Since $K_{2}:=\operatorname{supp} \hat{v} \Subset \mathbb{R}^{n}$ while $K_{1}:=\operatorname{supp} \hat{b}$ is closed in $\mathbb{R}^{2 n}$, the set $K$ on the right hand side of (1.11) is closed. Suppose first that $b$ is a Schwartz function and $\hat{v} \in C_{0}^{\infty}$. Then (1.10) holds. To avoid a cumbersome regularisation of $b$ with control of the spectra of the approximands, note that (1.10) also applies to $b_{\tau}(x, \eta):=b(x, \eta-\tau)$ for $\tau \in \mathbb{R}^{n}$. So with the partially reflected function $R \hat{b}_{\tau}(\xi, \eta)=\hat{b}(\xi, \tau-\eta)$, and $M=\left(\begin{array}{cc}I & 0 \\ -I & I\end{array}\right)$,

$$
\begin{aligned}
\mathcal{F}\left(b_{\tau}(x, D) v\right) & =\int R \hat{b}(\xi-\eta, \tau-\eta) \hat{v}(\eta) d \eta \\
& =R \hat{b} *\left(\left(\hat{v} \otimes \delta_{0}\right) \circ M\right)(\xi, \tau) .
\end{aligned}
$$

Since $\mathcal{S}\left(\mathbb{R}^{2 n}\right) \subset S_{1,0}^{d}\left(\mathbb{R}^{n} \times \mathbb{R}^{n}\right)$ is dense in the topology of $S_{1,0}^{d+1} \hookrightarrow \mathcal{S}^{\prime}\left(\mathbb{R}^{n}\right)$, and since the right hand side is in the set of convolutions $\mathcal{D}^{\prime} * \mathcal{E}^{\prime}$ on $\mathbb{R}^{2 n}$, it follows that (5.6) holds for $b \in S_{1,0}^{d}$, and then for all $\hat{v} \in \mathcal{E}^{\prime}\left(\mathbb{R}^{n}\right)$.

As functions of $\tau$, both sides are in $C\left(\mathbb{R}^{n}, \mathcal{D}^{\prime}\left(\mathbb{R}^{n}\right)\right) \subset \mathcal{D}^{\prime}\left(\mathbb{R}^{2 n}\right)$, for it is clear that the continuity with respect to $\tau$ of the symbol $b_{\tau} \in S_{1,0}^{d}$ is inherited by the left hand side. The right hand side of (5.6) has support in

$$
\tilde{K}=\left\{(\xi,-\theta)+(\eta, \eta) \mid(\xi, \theta) \in K_{1}, \eta \in K_{2}\right\}
$$

which is closed when $\hat{v} \subset \mathcal{E}^{\prime}$; and $\tilde{K} \cap\left(\mathbb{R}^{n} \times\{0\}\right)$ equals $K \times\{0\}$. So any $\varphi \in C_{0}^{\infty}\left(\mathbb{R}^{n}\right)$ with support in $\mathbb{R}^{n} \backslash K$ yields a positive distance from $\tilde{K}$ to $\operatorname{supp} \varphi \times\{0\}$; hence any $\psi \in C_{0}^{\infty}\left(\mathbb{R}^{n}\right)$ with $\int \psi=1$ will entail that, eventually, $\varphi(\xi) j^{n} \psi(j \tau)$ has support disjoint from $\tilde{K}$, hence by (5.5) that

$$
\begin{aligned}
0 & =\int\left\langle R \hat{b} *\left(\left(\hat{v} \otimes \delta_{0}\right) \circ M\right)(\cdot, \tau), \varphi\right\rangle j^{n} \psi(j \tau) d \tau \\
& =\int\left\langle\mathcal{F}\left(b_{\tau}(x, D) v\right), \varphi\right\rangle j^{n} \psi(j \tau) d \tau \\
& \underset{j \rightarrow \infty}{\longrightarrow}\langle\mathcal{F}(b(x, D) v), \varphi\rangle .
\end{aligned}
$$

Finally, for $b \in S_{1,1}^{\infty}$ it suffices to note that $b(x, D)$, according to the proof of Proposition 1.3, acts on $v$ as some operator with symbol in $S^{-\infty}$ for which the set $K$ is the same as for $b$. This completes the proof of Proposition 1.4. 
5.2. Proof of Corollary 1.2. Let for simplicity $u \in F_{p, q}^{s+d}$ and $q<\infty$. Mimicking (4.14) one finds

$$
\left\|\left(\sum_{k=2}^{\infty} 2^{s k q}\left|\sum_{j=0}^{k-2} a_{j, k}(x, D) u_{k}\right|^{q}\right)^{\frac{1}{q}}\right\|_{p} \leq c \mu_{0, n+1}(a)\left\|u \mid F_{p, q}^{s+d}\right\| .
$$

The conjunction of this estimate and the spectral property (S1) implies, by the first part of Lemma 2.1, that for $m=1$

$$
\left\|a^{(m)}(x, D) u\left|F_{p, q}^{s}\|\leq c\| u\right| F_{p, q}^{s+d}\right\|
$$

For $m=3$ one can analogously combine (S3) with a similar modification of (4.19), whereby $2^{-j N}$ gets replaced by $2^{j(s-N)}$, now for $N>s$.

Concerning $a^{(2)}$, it is easy to show in analogy with (4.14) that for the three possible combinations of $j, l$ one has

$$
\left\|\left(\sum_{k=2}^{\infty} 2^{s k q}\left|a_{k-j, k-l}(x, D) u_{k-l}\right|^{q}\right)^{\frac{1}{q}}\right\|_{p} \leq c \mu_{0, n+1}(a)\left\|u \mid F_{p, q}^{s+d}\right\| .
$$

In view of this, (S2) and the assumption $s>0$, the criterion for series with spectra in balls (cf Lemma 2.1) gives (5.10) for $m=2$.

Under the last assumption, (S2') yields for all large $k$ that the $k^{\text {th }}$ term in $a^{(2)}$ has spectrum in an annulus, so the criterion for such series and (5.11) apply; the remaining finitely many terms all lie in $\bigcap_{\sigma>0} F_{p, \infty}^{\sigma}$ by the first part of Lemma 2.1. Hence (5.10) holds for all $s \in \mathbb{R}$, all $m$.

Finally (1.9) is obtained analogously, and this completes the proof.

Remark 5.1. The mapping properties (1.8) and (1.9) were announced by Runst [18], albeit with somewhat flawed proofs: in connection with his Lemma 1 on the basic spectral estimates, there is [18, p. 20] an explicit appeal to a formula like (1.10) above, but this is not quite enough when symbols in $S_{1,1}^{\infty}$ and functions in, say $L_{1}^{\text {loc }} \backslash \mathcal{S}$ are treated simultaneously. The same flaw seems to be present in Marschall's work, for although the spectral properties are claimed in [13] without arguments, (1.10) was also appealed to in $[12$, p. 495]. However, these shortcomings are only of a technical nature, and they may easily be remedied by means of the support rule in Proposition 1.4, which has sufficiently weak assumptions.

\section{Final remarks}

The $B_{p, q}^{s}$ and $F_{p, q}^{s}$ spaces have for half a century been treated from many points of view, and it is known that they besides the $H_{p}^{s}$ also contain HölderZygmund classes and other function spaces. The books of Triebel [21, 22] 
account for this and describe the historic development and priorities. Here the names Besov and Triebel-Lizorkin spaces are used, since this seems to be common. Also the unifiying definition by means of Littlewood-Paley decompositions, cf Section 2, has been adopted for simplicity.

However, with these definitions, it is well known that the spaces make sense also for $p$ and $q$ in the interval ]0,1[ (although they are then only quasi-Banach spaces); hence it should be natural to give a brief treatment of these cases.

6.1. Exponents $p$ and $q$ in $] \mathbf{0}, \mathbf{1}[$. When extending the continuity results in Corollary 1.2 to $p, q$ in the full range $] 0, \infty]$, the first step could be to replace Lemma 2.1 by the well-known criteria in $[17,2.3 .2 / 2]$ or $[23$, Theorem 3.7], which both require that $s>\max \left(0, \frac{n}{p}-n, \frac{n}{q}-n\right)$. But there is a somewhat stronger result showing that the value of $q$ only matters for the "target space", while it is inconsequential for the mere convergence of the series:

Lemma 6.1. Let $s>\max \left(0, \frac{n}{p}-n\right)$ for $0<p<\infty$ and $0<q \leq \infty$ and suppose $u_{j} \in \mathcal{S}^{\prime}\left(\mathbb{R}^{n}\right)$ such that, for some $A>0$,

$$
\operatorname{supp} \mathcal{F} u_{j} \subset B\left(0, A 2^{j}\right), \quad F(q):=\left\|\left(\sum_{j=0}^{\infty} 2^{s j q}\left|u_{j}(\cdot)\right|^{q}\right)^{\frac{1}{q}}\right\|_{p}<\infty .
$$

Then $\sum_{j=0}^{\infty} u_{j}$ converges in $\mathcal{S}^{\prime}\left(\mathbb{R}^{n}\right)$ to some $u \in F_{p, r}^{s}\left(\mathbb{R}^{n}\right)$ for

$$
r \geq q, \quad r>\frac{n}{n+s},
$$

and then $\left\|u \mid F_{p, r}^{s}\right\| \leq c F(r)$ for some $c>0$ depending on $n, s, p$ and $r$.

Proof. If $s>\max \left(0, \frac{n}{p}-n, \frac{n}{q}-n\right)$, then $r=q$ is possible and the claim is just the usual result. Otherwise $q \leq \frac{n}{n+s}$, and $F(r) \leq F(q)<\infty$ for $r>q$; if also $r>\frac{n}{n+s}$ the standard result gives the statement.

Using this, Corollary 1.2 extends to $p$ and $q \in] 0, \infty]$ as follows:

Corollary 6.2. If $a \in S_{1,1}^{d}\left(\mathbb{R}^{n} \times \mathbb{R}^{n}\right)$ the corresponding operator $a(x, D)$ is bounded for $s>\max \left(0, \frac{n}{p}-n\right), 0<p, q \leq \infty$ and $r$ as in (6.2),

$$
\begin{aligned}
& a(x, D): F_{p, q}^{s+d}\left(\mathbb{R}^{n}\right) \rightarrow F_{p, r}^{s}\left(\mathbb{R}^{n}\right) \quad(p<\infty) ; \\
& a(x, D): B_{p, q}^{s+d}\left(\mathbb{R}^{n}\right) \rightarrow B_{p, q}^{s}\left(\mathbb{R}^{n}\right) .
\end{aligned}
$$

If (1.4) holds, then (6.3) and (6.4) do so for all $s \in \mathbb{R}$ and $r=q$.

Proof. The proof of Corollary 1.2 is easily carried over with the same relations as in $(\mathrm{S} 1)-(\mathrm{S} 3)$. The estimates are now made for $t \in] 0, \min (p, q)[$, 
so that (4.12) still applies (cf [23] eg); but taking $T>0$ such that $\frac{n}{t}<n+T$ it follows that $W_{1}^{n+T}$-estimates of the symbols suffice (they are controlled by the semi-norms $\mu_{0, n+T}(a)$ and $\left.\mu_{N, n+T}(a)\right)$.

Moreover (5.10) will need to have $q$ replaced by $r$ on the left hand side when Lemma 6.1 is invoked instead of Lemma 2.1. And when (1.4) holds, (S2') still applies, with spectra in annuli except for a finite part of $a^{(2)}(x, D) u$.

Remark 6.3. On the one hand, Corollary 6.2 improves [20] since the assumption $s>\max \left(0, \frac{n}{p}-n\right)$ is weaker than his $s>\max \left(0, \frac{n}{p}-n, \frac{n}{q}-n\right)$ (the latter is a well-known requirement in connection with Triebel-Lizorkin spaces, eg it occurs in many places in $[21,23,4,13,17]$, but it is avoided by the sharper statements in Lemma 6.1). And the condition (1.4) on the twisted diagonal has not been extended to the full range of $F_{p, q^{-}}^{s}$ and $B_{p, q^{-}}^{s}$ spaces before. On the other hand, $[6,7,20]$ also treat the continuity from a specific space $F_{p, q}^{s}$ with sufficient conditions of various kinds, even with some necessary conditions in $[6,7]$, cf also [8]; the reader is referred to these works for details.

Acknowledgement. My thanks are due both to prof. G. Grubb and to prof. V. Burenkov for their interest in this work.

\section{References}

[1] G. Bourdaud, Une algèbre maximale d'opérateurs pseudo-différentiels, Comm. Partial Differential Equations, 13 (9) (1988), 1059-1083.

[2] Chin Hung Ching, Pseudo-differential operators with nonregular symbols, J. Differential Equations, 11 (1972), 436-447.

[3] R. Coifman and Y. Meyer, Au delà des opérateurs pseudo-différentiels, Astérisque, vol. 57, Société Mathématique de France, Paris, 1978.

[4] M. Frazier and B. Jawerth, A discrete transform and decomposition of distribution spaces, J. Func. Anal., 93 (1990), 34-170.

[5] L. Hörmander, The Analysis of Linear Partial Differential Operators, Grundlehren der mathematischen Wissenschaften, Vol. 256, 257, 274, 275, Springer Verlag, Berlin, 1983, 1985.

[6] L. Hörmander, Pseudo-differential operators of type 1,1, Comm. Partial Differential Equations, 13 (9) (1988), 1085-1111.

[7] L. Hörmander, Continuity of pseudo-differential operators of type 1, 1 , Comm. Partial Differential Equations, 14 (2) (1989), 231-243. 
[8] L. Hörmander, Lectures on Nonlinear Differential Equations, Mathématiques \& applications, Vol. 26, Springer Verlag, Berlin, 1997.

[9] J. Johnsen, Traces of Besov spaces revisited, Z. Anal. Anwendungen, 19 (3) (2000), 763-779.

[10] J. Johnsen, Domains of type 1,1 operators: a case for Triebel-Lizorkin spaces, C. R. Acad. Sci. Paris Sér. I Math., 339 (2) (2004), 115-118.

[11] J. Marschall, Pseudo-Differential Operators with Non-Regular Symbols, Ph.D. thesis, Free University of Berlin, 1985.

[12] J. Marschall, Weighted parabolic Triebel spaces of product type, Fourier multipliers and pseudo-differential operators, Forum Math., 3 (5) (1991), 479-511.

[13] J. Marschall, Nonregular pseudo-differential operators, Z. Anal. Anwendungen, 15 (1) (1996), 109-148.

[14] Y. Meyer, Régularité des solutions des équations aux dérivées partielles non linéaires (d'après J.-M. Bony), Bourbaki Seminar, Vol. 1979/80, Lecture Notes in Math., Vol. 842, Springer, Berlin, 1981, pp. 293-302.

[15] Y. Meyer, Remarques sur un théorème de J.-M. Bony, Proceedings of the Seminar on Harmonic Analysis (Pisa, 1980), 1981, 1-20.

[16] Bui Huy Qui, On Besov, Hardy and Triebel spaces for $0<p \leq 1$, Ark. Mat., 21 (1983), 169-184.

[17] T. Runst and W. Sickel, Sobolev Spaces of Fractional Order, Nemytski Operators and Non-Linear Partial Differential Equations, Nonlinear Analysis and Applications, Vol. 3, de Gruyter, Berlin, 1996.

[18] T. Runst, Pseudodifferential operators of the "exotic" class $L_{1,1}^{0}$ in spaces of Besov and Triebel-Lizorkin type, Ann. Global Anal. Geom., 3 (1) (1985), 13-28.

[19] X. Saint Raymond, Elementary Introduction to the Theory of PseudoDifferential Operators, Studies in Advanced Mathematics, CRC Press, Boca Raton, FL, 1991.

[20] R. H. Torres, Continuity properties of pseudodifferential operators of type 1,1, Comm. Partial Differential Equations, 15 (1990), 1313-1328.

[21] H. Triebel, Theory of Function Spaces, Monographs in mathematics, Vol. 78, Birkhäuser Verlag, Basel, 1983.

[22] H. Triebel, Theory of Function Spaces II, Monographs in mathematics, Vol. 84, Birkhäuser Verlag, Basel, 1992.

[23] M. Yamazaki, A quasi-homogeneous version of paradifferential operators, I. Boundedness on spaces of Besov type, J. Fac. Sci. Univ. Tokyo Sect. IA, Math., 33 (1986), 131-174. 
Department of Mathematics

Aalborg University

Fredrik Bajers Vej 7G

DK-9220 Aalborg Øst

Denmark

(E-mail : jjohnsen@math.aau.dk)

(Received : September 2004) 


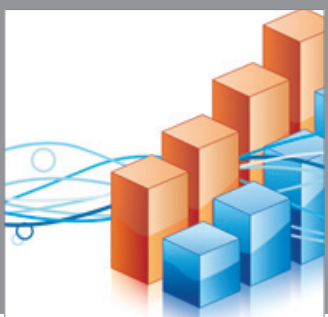

Advances in

Operations Research

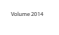

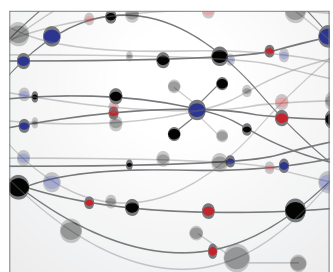

\section{The Scientific} World Journal
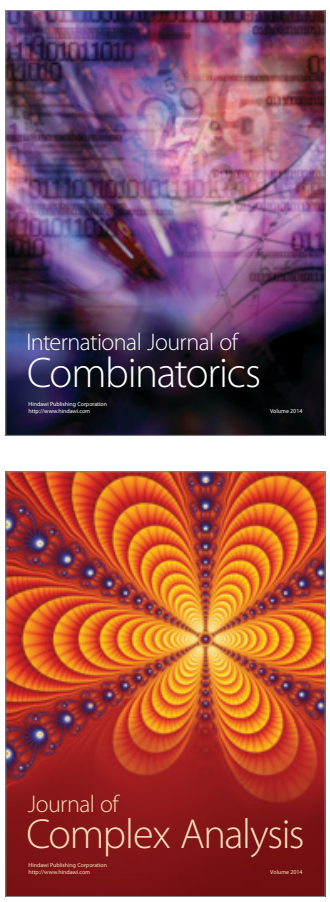

International Journal of

Mathematics and

Mathematical

Sciences
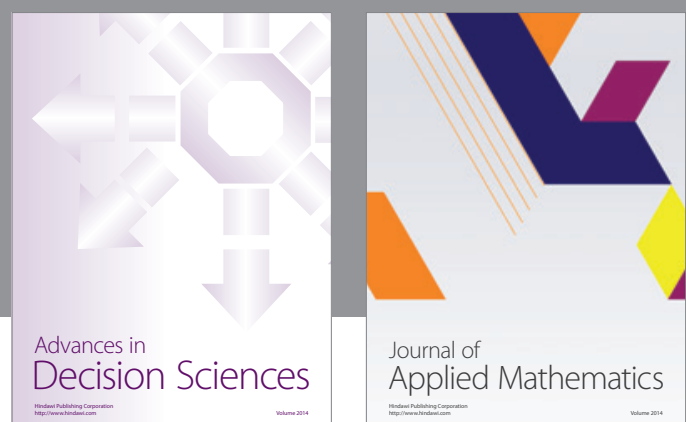

Journal of

Applied Mathematics
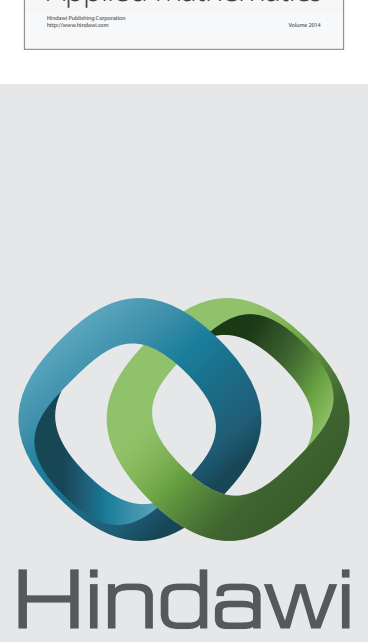

Submit your manuscripts at http://www.hindawi.com
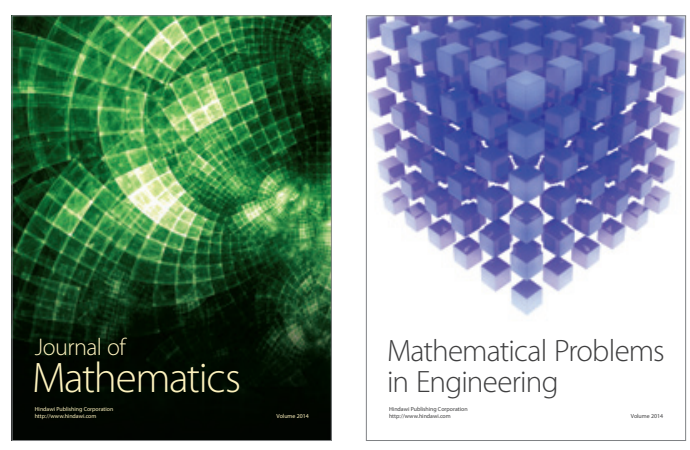

Mathematical Problems in Engineering
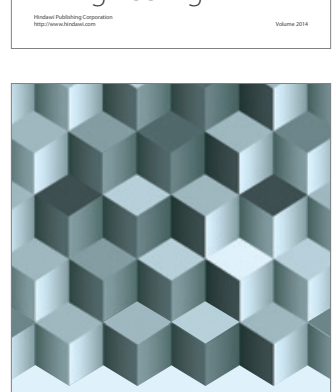

Journal of

Function Spaces
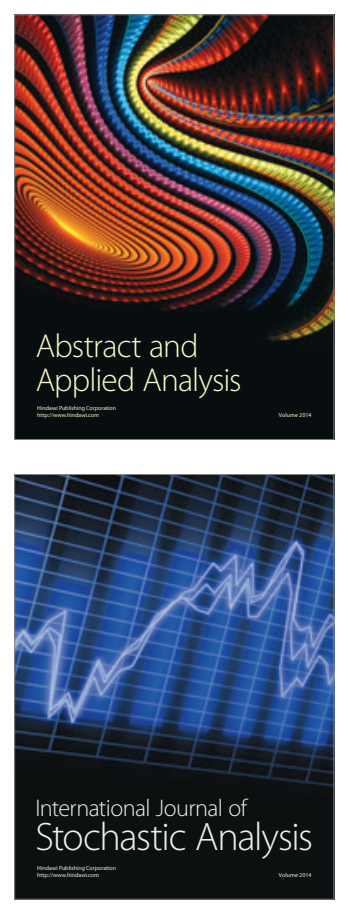

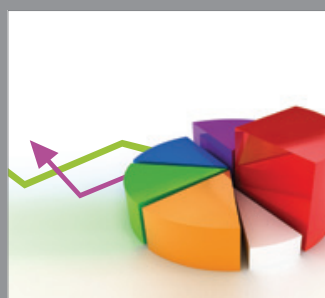

ournal of

Probability and Statistics

Promensencen
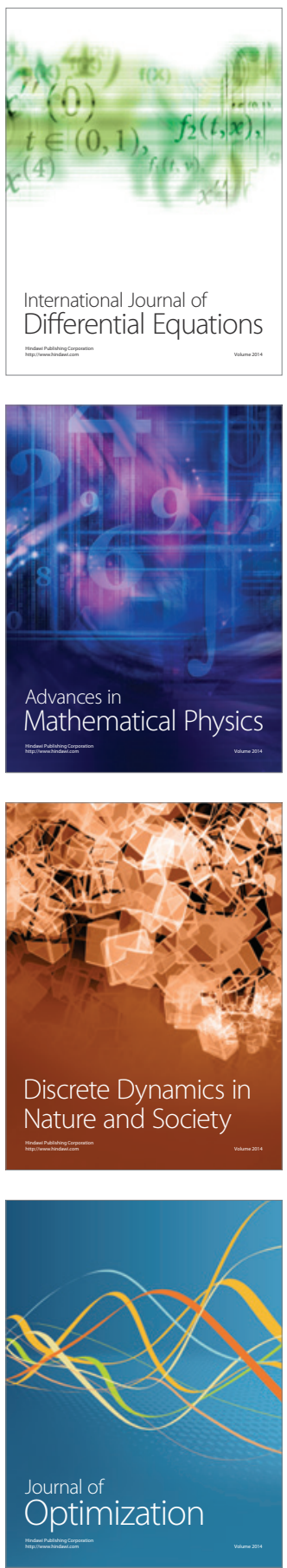